\title{
Editorial
}

\section{Subclinical Hypothyroidism and Pregnancy: The Intersection of Science, the Art of Medicine, and Public Health Policy}

\author{
Alex Stagnaro-Green \\ University of Illinois College of Medicine at Rockford, Rockford, III., USA
}

Pregnancy is a stress test for the thyroid [1]. In a pregnancy in which the thyroid gland has a normal physiological response, it maintains the euthyroid state by increasing the production of thyroxine-binding globulin, total thyroxine, and total triiodothyronine by as much as $50 \%$. The increased demand on the thyroid begins soon after conception, peaks early in the second trimester, and is maintained until delivery. The majority of women have normal thyroid reserve and therefore the thyroid responds adequately to the increased demands experienced during pregnancy. However, in women with decreased reserve, either due to autoimmune thyroid disease or iodine deficiency, subclinical hypothyroidism (SCH) develops during pregnancy despite the fact that the woman was euthyroid prior to conception. Estimates are that $2-3 \%$ of all pregnant women have undiagnosed $\mathrm{SCH}$, with some recent studies reporting a prevalence rate of over $10 \%$. Another subset of women prone to develop SCH are individuals who were on levothyroxine prior to pregnancy. These women cannot respond to the demands of pregnancy with enhanced endogenous thyroid hormone production and, in the majority of cases, will develop SCH during pregnancy unless their pre-pregnancy dose of levothyroxine is increased.
Research over the last 20 years has demonstrated a strong association between SCH and adverse outcomes in the mother, fetus and the offspring. With regard to the mother, $\mathrm{SCH}$ has been linked to gestational hypertension, gestational diabetes mellitus, and pre-eclampsia. Focusing on the fetus, $\mathrm{SCH}$ has been associated with an increase of spontaneous miscarriage, preterm delivery, and admission to the neonatal intensive care unit. Finally, studies have documented a decrease in the IQ of the offspring of mothers who had untreated SCH during delivery.

Given the prevalence of SCH and the recent appreciation of its association with negative outcomes experienced by the mother, fetus and offspring, in 2011 the American Thyroid Association (ATA) [2] and in 2012 the Endocrine Society (ES) [3] published clinical guidelines on thyroid and pregnancy. Both sets of guidelines cover the entire gamut of thyroid dysfunction during pregnancy and postpartum including hypothyroidism, hyperthyroidism, thyroid antibodies in euthyroid women, thyroid nodules, thyroid cancer and postpartum thyroiditis.

It is in the context of the rapidly evolving literature in the field of thyroid and pregnancy, in conjunction with the guidelines published over the last 3 years, that the guidelines developed by the European Thyroid Associa-

\begin{tabular}{ll}
\hline KARGER & $\begin{array}{l}\text { ○ 2014 European Thyroid Association } \\
\text { Published by S. Karger AG, Basel }\end{array}$ \\
$\begin{array}{l}\text { E-Mail karger@karger.com } \\
\text { www.karger.com/etj }\end{array}$ &
\end{tabular}

Alex Stagnaro-Green, MD, MHPE, Regional Dean

University of Illinois College of Medicine at Rockford

1601 Parkview Avenue

Rockford, IL 61101 (USA)

E-Mail alexsg@uic.edu 
tion (ETA) should be evaluated [4]. The ETA clinical guidelines, established by a distinguished panel of experts in the field of thyroid and pregnancy, and entitled 'Management of Subclinical Hypothyroidism in Pregnancy and in Children', are unique in two ways. First of all, they focus on the most controversial topic in the field of thyroid and pregnancy, namely whether or not $\mathrm{SCH}$ should be treated during pregnancy and its direct corollary, whether or not all pregnant women should be screened for SCH. Secondly, for the first time, they present a set of recommendations for the management of children with SCH.

After carefully reviewing the literature, the ETA committee generated 23 recommendations regarding $\mathrm{SCH}$ in pregnancy. The vast majority of recommendations are similar to those published by the prior guidelines. The most notable areas of difference center on the management of SCH and isolated hypothyroxinemia (normal $\mathrm{TSH}$ and decreased free $\mathrm{T}_{4}$ ). The ETA guidelines state that ' $\mathrm{SCH}$ arising preconception or during gestation should be treated with levothyroxine'. This is identical to the ES guidelines but differs from the ATA guidelines, which recommend treating SCH in women who are thyroid peroxidase positive but state that 'there is insufficient evidence to recommend for or against universal $\mathrm{LT}_{4}$ treatment in $\mathrm{TAb}$-pregnant women with $\mathrm{SCH}^{\prime}$. It should be noted that the majority of women with $\mathrm{SCH}$ during pregnancy are thyroid antibody negative. Similar differences exist with regard to the management of isolated hypothyroxinemia (IH). Whereas the ETA states that levothyroxine therapy should be considered in $\mathrm{IH}$, the ATA recommends against the treatment of $\mathrm{IH}$.

As the three guideline committees used the identical literature to formulate their evidence-based recommendations, how can such important differences exist? The answer lies in the intersection between science, the art of medicine, and the development of public health policy (which in this case is the creation of clinical guidelines). Focusing on the scientific evidence, multiple studies have shown an association between $\mathrm{SCH}$ during pregnancy and adverse outcomes, but of the two randomized controlled intervention trials performed to date, one demonstrated a benefit of treating SCH in women who were thyroid peroxidase antibody positive and the other found no benefit in treating pregnant women with SCH. With regard to $\mathrm{IH}$, the data in support of treatment are even weaker. The literature on demonstrating an association between IH and decreased IQ in the offspring is mixed and in the one intervention trial performed no benefit was found.
Herein comes the art of medicine. Everyday, clinicians make clinical decisions based on imperfect evidence. While aspiring to practice guided solely by well-developed evidence supported by multiple randomized controlled trials, the reality is that clinical medicine is most frequently practiced through the use of the best available evidence which is extrapolated to the clinical situation at hand. SCH is a perfect example for that. As noted, association studies have demonstrated a relationship between $\mathrm{SCH}$ and adverse pregnancy outcomes but only two prospective randomized trials have been performed and yielded opposite results. With regard to $\mathrm{IH}$, not only are the results of the association studies mixed, but the one prospective randomized intervention trial performed demonstrated no benefit in treating IH. Nevertheless, both the ETA and ES recommend treating $\mathrm{SCH}$ while the ATA recommend only treating $\mathrm{SCH}$ in thyroid peroxidase antibody women. With regard to IH, the ETA states that 'levothyroxine therapy may be considered in isolated hypothyroxinemia' while the ATA recommends that 'isolated hypothyroxinemia should not be treated in pregnancy'.

The reason for the discrepant recommendations is that insufficient data exist to make definitive recommendations and therefore the art of medicine impacts the conclusions. The thought process behind recommending treating SCH or not goes as follows. Proponents of treating SCH in all pregnant women conclude that, as levothyroxine has no side effects when prescribed at an appropriate dose, all women with $\mathrm{SCH}$ should be treated in order to potentially decrease the myriad of associated adverse pregnancy outcomes. On the other hand, proponents of no intervention would argue that since the data do not demonstrate a benefit of treating $\mathrm{SCH}$, levothyroxine intervention is not justifiable. Furthermore, therapy would result in the labeling of the mother with a disease causing her undue concern, and the fiscal impact on society of treating all women with SCH (at least 2-3\% of the pregnant population) would be substantial. Given this background it is easy to understand the varied conclusions reached by the various guideline committees. They reflect a different mix in the use of the science and art of medicine to create policy. Given the present state of knowledge, each of the societies' recommendations are valid in their own right. Most importantly, however, when counseling a pregnant woman with $\mathrm{SCH}$, she should be informed of the data so that she may partner with the clinician and make an informed decision.

The inclusion of a section on SCH in childhood and adolescence is a welcome addition to the guidelines on 
thyroid and pregnancy. The main recommendation, which is to treat children under 3 years of age who have TSH levels between 5.5 and $10.0 \mathrm{mU} / \mathrm{l}$ with levothyroxine in order to optimize brain development, should be universally adopted. As noted, after the age of 3 , a withdrawal trail should be attempted to ascertain whether the thyroid dysfunction is transient or permanent. Although the data on the negative impact of $\mathrm{SCH}$ on neuropsychological development, growth and obesity after the age of 3 are mixed, it is noteworthy that the studies on lipids and cardiac function showed a consistent negative impact. Further research in this area is clearly needed.

In conclusion, the ETA guidelines are thorough, thoughtful and focused on two important topics - the controversy surrounding the treatment of $\mathrm{SCH}$ during pregnancy and the impact of $\mathrm{SCH}$ in childhood and its management. The guidelines also appropriately stress the importance of iodine supplementation during pregnancy and lactation, as much of Europe remains mildly iodinedeficient. As noted in the introduction of the ETA guidelines, much variability exists in the management of thyroid disease during pregnancy. The ETA guidelines will need to be coupled with an aggressive education initiative focused on both clinicians and pregnant women in order to ensure that optimal care is being provided to all women.

\section{Disclosure Statement}

The author has no conflicts of interest to disclose.
References

Subclinical Hypothyroidism and Pregnancy
1 Shah MS, Davies TF, Stagnaro-Green A: The thyroid during pregnancy: a physiological stress test. Minerva Endocrinol 2003;28:233245.

-2 Stagnaro-Green A, Abalovich M, Alexander E, Azizi F, Mestman J, Negro R, Nixon A, Pearce EN, Soldin OP, Sullivan S, Wiersinga W: Guidelines of the American Thyroid Association for the diagnosis and management of thyroid disease during pregnancy and postpartum. Thyroid 2011;21:1-45.
3 DeGroot LD, Abalovich M, Alexander EK, Amino N, Barbour L, Cobin RH, Eastman CJ, Lazarus JH, Luton D, Mandel S, Mestman J, Rovet J, Sullivan S: Management of thyroid dysfunction during pregnancy and postpartum: an Endocrine Society clinical practice guideline. J Clin Endocrinol Metab 2012;97: 2543-2565.

4 Lazarus J, Brown RS, Daumerie C, Hubalewska-Dydejczyk A, Negro R, Vidya B: Management of subclinical hypothyroidism in pregnancy and in children. Eur Thyroid J, in press. 